Rapid Reviews COVID-19

\title{
Review 1: "Neurological manifestations associated with COVID-19: a nationwide registry"
}

Yi Tang ${ }^{1}$, Chenjian $\mathbf{L i}^{2}$

${ }^{1}$ Professor in Neurology, Department of Neurology, Xuanwu Hospital, Capital Medical University, National Clinical Research Center for Geriatric Diseases Beijing, China,

${ }^{2}$ Professor, School of Life Sciences, Peking University, Beijing, China

Published on: Aug 20, 2020

DOI: $10.21428 / 2 e 3983 f 5.95 a 6 c 65 e$

License: Creative Commons Attribution 4.0 International License (CC-BY 4.0). 


\section{$\underline{\text { RR:C19 Evidence Scale rating by reviewer: }}$}

- Reliable. The main study claims are generally justified by its methods and data. The results and conclusions are likely to be similar to the hypothetical ideal study. There are some minor caveats or limitations, but they would/do not change the major claims of the study. The study provides sufficient strength of evidence on its own that its main claims should be considered actionable, with some room for future revision.

$* * * * * * * * * * * * * * * * * * * * * * * * * * * * * * * * * * * * * * *$

\section{Review:}

This is a timely and important report on the neurological manifestations associated with COVID-19 based on data from a nationwide registry in France. The current retrospective study included 222 COVID-19 patients with de novo neurological manifestations from 46 centers.

The main findings included the broad spectrum of neurological manifestations associated with SARS-CoV-2 infection including COVID-19 associated encephalopathy (CAE), acute ischemic cerebrovascular syndrome (AICS), encephalitis, Guillain-Barré Syndrome (GBS), etc. The investigation was conducted by the brain imaging, CSF profiling and clinical course analyses. The authors also reported the effect of corticosteroid in patients with encephalitis and CAE, and the effect of IVIG in patients with GBS. Taken as a while, this is informative for future intervention studies. The following are some minor suggestions:

1) It would be meaningful if the authors could compare the clinical characteristics of their patient cohort with patient cohorts from other regions, such as in China and USA, to check whether different SARS-CoV-2 variants are associated with different clinical manifestations.

2) It will be more useful for the community if the authors provide more information about the patients who received treatment of steroid, IVIG or other specific treatment, including dosage, clinical course after treatment, and the effect of treatment on both neurological deficits and respiratory manifestations.

3) The authors reported that the median age of included patients was 65 years (IQR 5372), while they reported three young patients with a median age of 20 years (IQR 19- 
35) in the section "Acute benign lymphocytic meningitis". Are the three young patients from the same retrospective cohort?

4) In the section "Clinical course (table 2)", the authors reported that "Eleven out of 15 patients (73.3\%) with GBS had progressive weakness in both arms and legs that could be associated with sensory symptoms." By "associated with sensory symptoms", could the authors clarify whether they mean that the weakness in limbs is accompanied by sensory symptoms, or partially caused by sensory symptoms? 